\title{
Checklist dos Rotifera (Animalia) do Estado de Mato Grosso do Sul, Brasil
}

\author{
Kennedy Francis Roche' \& William Marcos da Silva²
}

\begin{abstract}
1. Centro de Ciências Exatas e de Tecnologia, Universidade Federal de Mato Grosso do Sul, 79070-900 Campo Grande, MS, Brasil. (kennedy.roche@ufms.br) 2. Centro Universitário de Corumbá, Universidade Federal de Mato Grosso do Sul, 79304-902, Corumbá, MS, Brasil.
\end{abstract}

Recebido 1 dezembro 2016

Aceito 6 fevereiro 2017

DOI: $10.1590 / 1678-4766 e 2017105$

ABSTRACT. Checklist of the Rotifera (Animalia) from Mato Grosso do Sul State, Brazil. This study comprises a list of rotifer taxa found in the state of Mato Grosso do Sul, gleaned from the scientific literature. The studies were divided in five general areas: (1) the River Paraguay, (2) the Pantanal of Nhecolândia, (3) the rivers Miranda, Abobral and Itaqueri, (4) a reservoir in the city of Campo Grande, and (5) rivers, lakes and canals of the River Paraná. In total, 364 taxa were registered, with the families Lecanidae, Trichocercidae and Brachionidae showing greatest taxonomic richness, with 72 , 32 and 30 taxa, respectively. The greatest taxon richness was found in the area of the River Parana (244 taxa), followed by the River Paraguay in the region of the city of Corumbá (with 154 taxa), and the freshwater lakes of Nhecolândia (with 148 taxa). This pattern is most probably due to the quantity and quality of the studies carried out in these areas.

KEYWORDS. Biodiversity, freshwater zooplankton, Alto Paraguay River Basin, Paraná River basin, Biota-MS Program.

RESUMO. Este estudo listou os táxons de Rotifera encontrado da literatura científica no estado de Mato Grosso do Sul. O estudo foi dividido em cinco áreas gerais: (1) rio Paraguai, (2) Pantanal de Nhecolândia, (3) rio Miranda, rio Negro, rio Abobral e Itaqueri, (4) represa Lago do Amor na cidade de Campo Grande, e (5) rios, lagos e canais do rio Paraná. Foram registrados um total de 364 táxons, sendo as famílias Lecanidae, Trichocercidae e Brachionidae as mais ricas, com 72, 32 e 30 táxons respectivamente. A maior riqueza de táxons foi registrada na área do rio Paraná (244 táxons), e em seguida no rio Paraguai na região da cidade de Corumbá (com 154 táxons encontrados), e nos lagos de água doce do Pantanal da Nhecolândia (com 148 táxons). É altamente provável que estes resultados são reflexos da quantidade e qualidade dos estudos nestas áreas.

PALAVRAS-CHAVE. Biodiversidade, zooplancton de água doce, Bacia do Alto Paraguai, Bacia do rio Paraná, Programa Biota-MS.

Os rotíferos são animais geralmente microscópicos, quase exclusivamente de água doce e geralmente abundantes em lagos, represas e rios, e também em solos úmidos. Tem grande importância nas teias alimentares de ambientes aquáticos, se alimentando de algas, protozoários, bactérias e detritos, servindo como presas para predadores invertebrados, bem como peixes, especialmente formas jovens e pequenas. Podem ter papéis muito importantes na reciclagem de nutrientes e serem úteis como indicadores de qualidade ambiental, na ecotoxicologia, e na aquicultura. O filo consiste de três grupos, o pequeno grupo marinho Seisonacea, e os grupos maiores de água doce, Bdelloidea e Monogononta. Em Bdelloidea não foram registrados machos, enquanto em Monogononta os mesmos são extremamente raros. Assim, a taxonomia é baseada quase exclusivamente das características das fêmeas. Para uma introdução à biologia, ecologia e sistemática do Rotifera, ver WALlace et al. (2006).

Nas ações de conservação e gestão da biodiversidade, estudos taxonômicos são de extrema importância (SEGERS, 2008). Para se ter uma visão do estado do conhecimento taxonômico em uma região e apontar lacunas na informação e direções de futuras pesquisas, os inventários taxonômicos são fundamentais (OliveIRA-NETO \& Moreno, 1999; SouZASoARES et al., 2011; GARRAFOni \& LourenÇO, 2012). No presente estudo, pela primeira vez, um inventário taxonômico do grupo Rotifera é compilado para o Estado de Mato Grosso do Sul.

\section{MATERIAL E MÉTODOS}

Para a compilação da lista de táxons de rotíferos encontrados no Estado de Mato Grosso do Sul foram utilizados artigos da literatura científica, bem como uma dissertação de mestrado, uma tese de doutorado e relatórios não publicados. Táxons não reconhecidos por SEGERS (2007) são indicados na tabela da listagem; este trabalho é considerado como a mais correta revisão moderna, porque segue rigorosamente as regras do Código Internacional de Nomenclatura Zoológica (ICZN, 1999).

\section{RESULTADOS E DISCUSSÃO}

Os estudos foram divididos em cinco áreas (Tab. I), sendo o rio Paraguai, incluindo lagoas marginais e áreas 
inundadas, lagos de água doce e hiposalina do Pantanal da Nhecolândia, corpos de água associados com os rios Miranda, Negro, Abobral e Itaqueri, a represa Lago do Amor na cidade de Campo Grande, e rios, lagos e canais do rio Paraná.

Devido ao número reduzido de estudos e diferenças no esforço de amostragem (número de estudos, número de amostras analisadas), não é possível obter nenhuma conclusão em relação a padrões de biodiversidade de Rotifera no estado, em nível regional (OliveIrA-Neto \& Moreno, 1999; SouZASOAREs et al., 2011) ou em nível nacional (GARRAFONI \& LOURENÇO, 2012). A maior riqueza foi registrada na área do rio Paraná, seguida do rio Paraguai na região da cidade de Corumbá, e nos lagos de água doce da Nhecolândia (Tab. II). É provável que estes resultados refletem principalmente a quantidade e qualidade de pesquisa nestas áreas, o rio Paraná sendo pesquisado detalhadamente por vários anos (LANSACTôHA et al., 2009), e os outros dois estudos beneficiados por estudos do taxonomista Walter Koste (Koste, 1978). Assim, o efeito esforço/especialista seria a explicação para estes elevados valores de riqueza (Fontaneto et al., 2012). Podese concluir que mais estudos taxonômicos e ecológicos são necessários e urgentes, especialmente na região do Pantanal, uma Reserva da Biosfera e Patrimônio da Humanidade da UNESCO.

Quando amostras fixadas são analisadas, muitas formas de rotíferos, especialmente os aloricados sem apêndices distintos (sendo principalmente formas não planctônicas como membros das famílias de Notommatidae e Dicranophoridae, e o grupo Bdelloidea) não podem ser identificados. Assim, para identificação confiável e descrição de novas espécies, é necessário examinar organismos vivos. No Brasil, onde é frequentemente necessário viajar grandes distâncias para amostrar áreas de interesse longe de laboratórios, tais análises são muitas vezes inviáveis. A utilização de anestesia, por exemplo Bupivacaine (NoGRADY \& Rowe, 1993), ou água carbonificada pode ser útil, mas nem sempre funciona eficientemente.

A identificação de rotíferos, especialmente os aloricados, frequentemente depende de análises da microestrutura do mástax (mandíbula) (OBERTEGGER et al., 2006). Tais análises são frequentemente viáveis com o uso de microscopia convencional (óptica). Porém, o uso de microscopia eletrônica pode revelar mais detalhes, com maior confiabilidade.

A técnica de microscopia eletrônica é de grande utilidade em análises de microcaracterísticas de rotíferos (CLÉMENT \& WuRdaK, 1991), principalmente Microscopia Eletrônica de Varredura (SEM) aplicada ao mástax (DE SMET, 1998), sendo usada cada vez mais para a identificação de espécies (De Smet, 2005). MARKevich \& Kutikova (1989) propõem uma classificação nova de Rotifera, baseada inteiramente em estudos da ultraestrutura do mástax utilizando SEM. SANOAMUANG (1993b) e SHIEL \& SANOAMUANG (1993) utilizaram SEM para distinguir, na base da ultraestrutura do mástax, espécies de Filinia. Por exemplo, para distinguir a $F$. teminalis (uma espécie de água temperada) de $F$. novaezealandiae (uma espécie de água tropical) (SEGERS et al., 1996), é necessário contar o número de dentes do único do mástax; isto só pode ser feito com confiabilidade utilizando SEM. A frequente indisponibilidade

Tab. I. Identificação das localidades de amostragem de Rotifera, com coordenadas geográficas e referências dos estudos, Mato Grosso do Sul., Brasil.

\begin{tabular}{|c|c|c|}
\hline Código e localidade & Coordenadas (S-W) & Referência \\
\hline \multicolumn{3}{|l|}{ RIO PARAGUAI } \\
\hline 1. Lagoa Albuquerque & $19^{\circ} 10^{\prime} 30^{\prime \prime}-57^{\circ} 10^{\prime} 30^{\prime \prime}$ & EsPíNDOLA et al., 1996 \\
\hline 2. Corumbá; Canal do Tamengo & $18^{\circ} 59^{\prime} 31^{\prime \prime}-57^{\circ} 38^{\prime} 14.8^{\prime \prime}$ & $\begin{array}{l}\text { Koste, 1999; W. M. } \\
\text { Silva Observ. (comun. pess.) }\end{array}$ \\
\hline 3. Baia de Castelo & $18^{\circ} 34^{\prime} 38^{\prime \prime}-57^{\circ} 34^{\prime} 00^{\prime \prime}$ & Koste, 1999; Calheiros, 2003 \\
\hline 4. Rio Paraguai (37 localidades entre Porto Cáceres até o rio Apa) & $16^{\circ} 03^{\prime}-26^{\circ} 23^{\prime}$ até $22^{\circ} 03^{\prime}-58^{\circ} 00^{\prime}$ & FRUTOS et al., 2006 \\
\hline \multicolumn{3}{|l|}{ PANTANAL DE NHECOLÂNDIA } \\
\hline 5. Fazenda Nhumirim, lagos de água doce ("Baias") & $18^{\circ} 59^{\prime}-56^{\circ} 39^{\prime}$ & Koste, 1999; Medina JúNIOR, 1999 \\
\hline $\begin{array}{l}\text { 6. Fazenda Nhumirim; lago intermediário entre água doce e hiposa- } \\
\text { lina ("Salitrada") }\end{array}$ & & MEDINA JÚNIOR, 1999 \\
\hline $\begin{array}{l}\text { 7. Fazenda Nhumirim, lagos hiposalinos (“Salinas") Rios Miranda/ } \\
\text { Negro/Abobral/Itaqueri }\end{array}$ & & Koste, 1999; SiLva et al., 2010 \\
\hline 8. Fazenda Boa Sorte baia & $19^{\circ} 22^{\prime}-57^{\circ} 02^{\prime}$ & BONECKER et al., 1998 \\
\hline 9. Caixas de empréstimo, Passo do Lontra & & BONECKER et al., 1998 \\
\hline 10. Baia de Medalha, Passo do Lontra & $19^{\circ} 34^{\prime} 38^{\prime \prime}-57^{\circ} 01^{\prime} 05^{\prime \prime}$ & BONECKER et al., 1998 \\
\hline 11. Rio Miranda, Passo do Lontra & & BONECKER et al., 1998 \\
\hline 12. Rios Miranda, Abobral, Itaqueri & & OLIVEIRA-Neto, 1991 \\
\hline \multicolumn{3}{|l|}{ CAMPO GRANDE } \\
\hline 13. Lago do Amor & $20^{\circ} 30^{\prime} 12,1^{\prime \prime}-54^{\circ} 37^{\prime} 02^{\prime \prime}$ & Roche, 2010 \\
\hline \multicolumn{3}{|l|}{ RIO PARANÁ } \\
\hline 14. Represa, cidade de Dourados & $22^{\circ} 12^{\prime}-54^{\circ} 54^{\prime}$ & MARTÍNEZ et al., 2000 \\
\hline $\begin{array}{l}\text { 15. Rios Baía, Ivinheima, Paraná; lagos Guaraná, Patos, Fechada, } \\
\text { Pousada das Garças; canal Curutuba }\end{array}$ & $22^{\circ} 43^{\prime}-53^{\circ} 17^{\prime}$ & $\begin{array}{l}\text { BONECKER et al., 1994; } \\
\text { LANSAC-TÔHA et al., 1997; } \\
\text { GARCIA et al., 1998; } \\
\text { SERAFIM et al., 2003; } \\
\text { JOKO et al., 2008; } \\
\text { BONECKER et al., 2009; } \\
\text { LANSAC-TÔHA et al., } 2009\end{array}$ \\
\hline
\end{tabular}


Tab. II. Lista dos táxons encontrados nas localidades do Mato Grosso do Sul, Brasil (Tab. I). Táxons não reconhecidos por SEGERS (2007) são indicados pelo*.

\begin{tabular}{|c|c|c|c|c|c|c|c|c|c|c|c|c|c|c|c|}
\hline Táxon & 1 & 2 & 3 & 4 & 5 & 6 & 7 & 8 & 9 & 10 & 11 & 12 & 13 & 14 & 15 \\
\hline DIGONONTA (=BDELLOIDEA) & & & & & & & & & & & & & & & \\
\hline HABROTROCHIDAE & & & & & & & & & & & & & & & \\
\hline Habrotrocha angusticollis (Murray, 1905) & & $X$ & $X$ & & X & & & & & & & & & & \\
\hline PHILODINIDAE & & & & & & & & & & & & & & & \\
\hline Dissotrocha aculeata (Ehrenberg, 1832) & & & & & & $\mathrm{X}$ & & & & $\mathrm{X}$ & $\mathrm{X}$ & & & & $\mathrm{X}$ \\
\hline D. aculeata aculeata (Ehrenberg, 1832) & & $\mathrm{X}$ & & & & & & & & & & & & & \\
\hline $\begin{array}{l}\text { *D. aculeata björki (Berzins, 1982) (= } \\
\text { Dissotrocha bjoerki Bērzin̦š, 1982) }\end{array}$ & & $\mathrm{X}$ & & & & & & & & & & & & & \\
\hline D. aculeata inserta Bērziṇš, 1982 & & $\mathrm{X}$ & & & $\mathrm{X}$ & & & & & & & & & & \\
\hline *D. macrodactyla (Ehrenberg, 1838) & & & & & & & & & & & & & & & $X$ \\
\hline D. macrostyla (Ehrenberg, 1838) & & & & & $\mathrm{X}$ & $X$ & & & & & & & & & $\mathrm{X}$ \\
\hline D. schlienzi Hauer, 1956 & & & $\mathrm{X}$ & & $\mathrm{X}$ & & & & & & & & & & $\mathrm{X}$ \\
\hline Philodina megalotrocha Ehrenberg, 1832 & & $\mathrm{X}$ & $\mathrm{X}$ & & $\mathrm{X}$ & & & & & & & & & & \\
\hline P. rugosa Bryce, 1903 & & & & & $\mathrm{X}$ & & & & & & & & & & \\
\hline Pleuretra brycei (Weber, 1898) & & & & & $\mathrm{X}$ & & & & & & & & & & \\
\hline P. humerosa (Murray, 1905) & & $\mathrm{X}$ & & & $\mathrm{X}$ & & & & & & & & & & \\
\hline Rotaria neptunia (Ehrenberg, 1830) & & $\mathrm{X}$ & $\mathrm{X}$ & & & $\mathrm{X}$ & & & & & & & & $\mathrm{X}$ & $\mathrm{X}$ \\
\hline R. rotatoria (Pallas, 1766) & & $\mathrm{X}$ & $\mathrm{X}$ & & $\mathrm{X}$ & & & & & & & & & & $\mathrm{X}$ \\
\hline R. sordida (Western, 1893) & & $\mathrm{X}$ & & & & & & & & & & & & & $\mathrm{X}$ \\
\hline R. tardigrada (Ehrenberg, 1830) & & $\mathrm{X}$ & $\mathrm{X}$ & & & & & & & & & & & $\mathrm{X}$ & $\mathrm{X}$ \\
\hline MONOGONONTA & & & & & & & & & & & & & & & \\
\hline ASPLANCHNIDAE & & & & & & & & & & & & & & & \\
\hline Asplanchna brightwelli Gosse, 1850 & $X$ & $X$ & & & & & & & & & & & & & \\
\hline Asplanchna priodonta Gosse, 1850 & & & & & & & & & & & & & & & $\mathrm{X}$ \\
\hline Asplanchna sieboldi (Leydig, 1854) & & $\mathrm{X}$ & & & & & & & & & & $\mathrm{X}$ & & & $\mathrm{X}$ \\
\hline Harringia rousseleti de Beauchamp, 1912 & & $\mathrm{X}$ & & & & & & & & & & & & & \\
\hline ATROCHIDAE & & & & & & & & & & & & & & & \\
\hline Cupelopagis vorax (Leidy, 1857) & & $\mathrm{X}$ & $\mathrm{X}$ & & & & & & & & & & & & \\
\hline BRACHIONIDAE & & & & & & & & & & & & & & & \\
\hline Anuraeopsis fissa Gosse, 1851 & & $\mathrm{X}$ & $\mathrm{X}$ & & & & & & & & & & & & $\mathrm{X}$ \\
\hline Anuraeopsis navicula Rousselet, 1911 & $\mathrm{X}$ & & & & & & & & & & & & & & \\
\hline Brachionus angularis Gosse, 1851 & & $\mathrm{X}$ & $\mathrm{X}$ & & $\mathrm{X}$ & $\mathrm{X}$ & $\mathrm{X}$ & & & & & & & & $\mathrm{X}$ \\
\hline B. bidentatus Anderson, 1889 & & & & & & & & & & & & $\mathrm{X}$ & & & \\
\hline B. budapestinensis Daday, 1885 & & & & & & & & & & & & & & & $X$ \\
\hline B. calyciflorus Pallas, 1766 & & & $\mathrm{X}$ & & & & & & & & & & $\mathrm{X}$ & $\mathrm{X}$ & $\mathrm{X}$ \\
\hline *B. calyciflorus f. spinosus Rousselet, 1901 & & & & & & & & & & & & & & & $\mathrm{X}$ \\
\hline B. caudatus Barrois \& Daday, 1894 & $\mathrm{X}$ & $\mathrm{X}$ & & $\mathrm{X}$ & & & & & & & & $\mathrm{X}$ & & & $\mathrm{X}$ \\
\hline *B. caudatus f. austrogenitus Ahlstrom, 1940 & & & & & & & & & & & & & & & $\mathrm{X}$ \\
\hline * B. caudatus f. insuetus Ahlstrom, 1940 & $\mathrm{X}$ & $\mathrm{X}$ & & & & & & & & & & & & & $\mathrm{X}$ \\
\hline *B. caudatus f. majusculus Ahlstrom, 1940) & & & & & & & & & & & & & & & $\mathrm{X}$ \\
\hline $\begin{array}{l}\text { *B. caudatus f. personatus Ahlstrom } 1940 \\
\text { (= Brachionus ahlstromi Lindeman, 1939) }\end{array}$ & $\mathrm{X}$ & $\mathrm{X}$ & & $\mathrm{X}$ & & & & & & & & & & & $\mathrm{X}$ \\
\hline *B. chelonis & & $\mathrm{X}$ & & & & & & & & & & & & & \\
\hline B. dimidiatus f. inermis Bryce, 1931 & & $\mathrm{X}$ & & & $\mathrm{X}$ & & $\mathrm{X}$ & & & & & & & & \\
\hline B. dolobratus Harring, 1914 & $\mathrm{X}$ & $\mathrm{X}$ & & & $\mathrm{X}$ & & & & & & & $\mathrm{X}$ & $\mathrm{X}$ & $\mathrm{X}$ & $\mathrm{X}$ \\
\hline B. falcatus Zacharias, 1898 & $\mathrm{X}$ & $\mathrm{X}$ & $\mathrm{X}$ & $\mathrm{X}$ & $\mathrm{X}$ & & & & & & & $\mathrm{X}$ & $\mathrm{X}$ & $\mathrm{X}$ & $\mathrm{X}$ \\
\hline *B. forficula forficula Wierzejski, 1891 & & & & & & & & & & & & & & & $\mathrm{X}$ \\
\hline B. havanaensis Rousselet, 1911 & & & & & & & & & & & & & $\mathrm{X}$ & & \\
\hline $\begin{array}{c}\text { *B. mirabilis Daday, } 1897 \text { (= Brachionus } \\
\text { quadridentatus mirabilis Daday, 1897) }\end{array}$ & & $X$ & $\mathrm{X}$ & & $\mathrm{X}$ & & & & & & & & & & \\
\hline B. mirus Daday, 1905 & & $\mathrm{X}$ & & & & & & & & & & $\mathrm{X}$ & & & $\mathrm{X}$ \\
\hline $\begin{array}{l}\text { * } B \text {. mirus angustus Koste, } 1972 \text { (= Brachionus } \\
\text { mirus Daday, 1905) }\end{array}$ & $\mathrm{X}$ & $\mathrm{X}$ & & $\mathrm{X}$ & & & & & & & & & & & $\mathrm{X}$ \\
\hline *B. mirus f. laticaudatus Paggi, 1973 & & $\mathrm{X}$ & & & & & & & & & & & & & \\
\hline $\begin{array}{l}\text { *B. mirus reductus Koste, } 1972 \text { (= Brachionus } \\
\text { mirus Daday, 1905) }\end{array}$ & & & & & $\mathrm{X}$ & & & & & & & & & & $\mathrm{X}$ \\
\hline B. quadridentatus Hermann, 1783 & $\mathrm{X}$ & $\mathrm{X}$ & & & $\mathrm{X}$ & $\mathrm{X}$ & & & & & & & & & $\mathrm{X}$ \\
\hline B. quadridentatus quadridentatus Hermann, 1783 & & & & & & & & & & & & $\mathrm{X}$ & & & $\mathrm{X}$ \\
\hline $\begin{array}{l}\text { B. quadridentatus melhemi Barrois \& Daday, } \\
1894\end{array}$ & & $\mathrm{X}$ & $\mathrm{X}$ & & & & & & & & & & & & $\mathrm{X}$ \\
\hline B. quadridentatus mirabilis Daday, 1897 & & & $\mathrm{X}$ & & & & & & & & & & & & $\mathrm{X}$ \\
\hline B. rubens Ehrenberg, 1838 & & & & & & & & & & & & & & & \\
\hline B. satanicus Rousselet, 1913 & & & & & & & & & & & & & & & $\mathrm{X}$ \\
\hline B. urceolaris Müller, 1773 & & & & & & & & & & & & & & & $\mathrm{X}$ \\
\hline $\begin{array}{l}\text { *B. urceolaris amazonica Koste \& Robertson, } \\
1983\end{array}$ & & & & & & & & & & & & & & & $\mathrm{X}$ \\
\hline
\end{tabular}


Tab. II. Cont.

\begin{tabular}{|c|c|c|c|c|c|c|c|c|c|c|c|c|c|c|c|}
\hline Táxon & 1 & 2 & 3 & 4 & 5 & 6 & 7 & 8 & 9 & 10 & 11 & 12 & 13 & 14 & 15 \\
\hline *B. cf. urceus (Linnaeus, 1758) & & & & & & & & & & & & & & & $\mathrm{X}$ \\
\hline Kellicottia bostoniensis (Rousselet, 1908) & & & & $\mathrm{X}$ & & & & & & & & & $\mathrm{X}$ & & $\mathrm{X}$ \\
\hline Keratella americana Carlin, 1943 & $\mathrm{X}$ & $\mathrm{X}$ & & $\mathrm{X}$ & & & & & & & & $\mathrm{X}$ & & $\mathrm{X}$ & $\mathrm{X}$ \\
\hline *K. americana hispida Lauterborn, 1898 & & $\mathrm{X}$ & & $\mathrm{X}$ & $\mathrm{X}$ & & & & & & & & & & \\
\hline K. cochlearis (Gosse, 1851$)$ & $\mathrm{X}$ & $\mathrm{X}$ & & $\mathrm{X}$ & & & & & & & & $\mathrm{X}$ & $\mathrm{X}$ & $\mathrm{X}$ & $\mathrm{X}$ \\
\hline K. cochlearis cochlearis (Gosse, 1851) & & $\mathrm{X}$ & & & & & & & & & & & & & $\mathrm{X}$ \\
\hline $\begin{array}{l}\text { *K. cochlearis f. macracantha (Lauterborn, } \\
1900)\end{array}$ & & & & & & & & & & & & & & & $\mathrm{X}$ \\
\hline $\begin{array}{l}\text { *K. cochlearis var. tecta f. micracantha } \\
\text { (Lauterborn, 1900) }\end{array}$ & & & & & & & & & & & & & & & $\mathrm{X}$ \\
\hline *K. cochlearis var. robusta (Lauterborn, 1900) & & $\mathrm{X}$ & & & $\mathrm{X}$ & & & & & & & & & & \\
\hline K. lenzi Hauer, 1953 & $\mathrm{X}$ & $\mathrm{X}$ & $\mathrm{X}$ & & $\mathrm{X}$ & $\mathrm{X}$ & & & & & & $\mathrm{X}$ & & & $\mathrm{X}$ \\
\hline K. paludosa (Lucks, 1912) & $\mathrm{X}$ & & & & & & & & & & & & & & \\
\hline K. tropica (Apstein, 1907) & & & & & $\mathrm{X}$ & $\mathrm{X}$ & & & & & & $\mathrm{X}$ & $\mathrm{X}$ & $\mathrm{X}$ & $\mathrm{X}$ \\
\hline *K. tropica tropica (Apstein, 1907) & & $\mathrm{X}$ & & $\mathrm{X}$ & $\mathrm{X}$ & & & & & & & & & & \\
\hline *K. tropica f. aspina (Fadeew, 1927) & & $\mathrm{X}$ & & & & & & & & & & & & & \\
\hline *K. tropica f. taurocephala Koste, 1978 & & $\mathrm{X}$ & & & & & & & & & & & & & \\
\hline K. valga (Ehrenberg, 1834) & $\mathrm{X}$ & $\mathrm{X}$ & & & & & & & & & & & & & \\
\hline Notholca acuminata (Ehrenberg, 1832) & & & & & & & & & & & & & & & $\mathrm{X}$ \\
\hline Plationus patulus (Müller, 1786) & $\mathrm{X}$ & & & & $\mathrm{X}$ & $\mathrm{X}$ & & & & & & & & & $\mathrm{X}$ \\
\hline P. patulus patulus (Müller, 1786) & & $\mathrm{X}$ & & & $\mathrm{X}$ & & & & & & & $\mathrm{X}$ & & & $\mathrm{X}$ \\
\hline P. patulus macracanthus (Daday, 1905) & & $\mathrm{X}$ & $\mathrm{X}$ & & $\mathrm{X}$ & & & & & & & & & & \\
\hline *P. macracanthus (Daday, 1905) & & & & & & $\mathrm{X}$ & & & & & & & & $\mathrm{X}$ & $\mathrm{X}$ \\
\hline Platyias leloupi Gillard, 1957 & & $\mathrm{X}$ & & & $\mathrm{X}$ & & & & & $\mathrm{X}$ & $\mathrm{X}$ & & & & $\mathrm{X}$ \\
\hline P. quadricornis (Ehrenberg, 1832) & $\mathrm{X}$ & $\mathrm{X}$ & $\mathrm{X}$ & & $\mathrm{X}$ & $\mathrm{X}$ & & & & & & $\mathrm{X}$ & & & $\mathrm{X}$ \\
\hline *P. quadricornis var. brevispinus Daday, 1905 & & & & & & & & & & $\mathrm{X}$ & $\mathrm{X}$ & & & & $\mathrm{X}$ \\
\hline P. quadricornis quadricornis (Ehrenberg, 1832) & & $\mathrm{X}$ & $\mathrm{X}$ & & $\mathrm{X}$ & & & $\mathrm{X}$ & & $\mathrm{X}$ & $\mathrm{X}$ & & & & $\mathrm{X}$ \\
\hline COLLOTHECIDAE & & & & & & & & & & & & & & & \\
\hline Collotheca campanulata (Dobie, 1849) & & & & & $\mathrm{X}$ & & & & & & & & & & \\
\hline Collotheca ornata (Ehrenberg, 1832) & & & $\mathrm{X}$ & & $\mathrm{X}$ & & & & & & & & & & $\mathrm{X}$ \\
\hline Collotheca tenuilobata (Anderson, 1889) & & $\mathrm{X}$ & & & & & & & & & & & & & \\
\hline CONOCHILIDAE & & & & & & & & & & & & & & & \\
\hline Conochilus coenobasis (Skorikov, 1914) & $\mathrm{X}$ & $X$ & & $\mathrm{X}$ & $X$ & & & & & & & $\mathrm{X}$ & $X$ & $\mathrm{X}$ & $\mathrm{X}$ \\
\hline Conochilus dossuarius Hudson, 1885 & & $X$ & $\mathrm{X}$ & & & & & & & & & & & & $\mathrm{X}$ \\
\hline Conochilus natans (Seligo, 1900) & & $\mathrm{X}$ & & & $\mathrm{X}$ & & & & & & & & & & $\mathrm{X}$ \\
\hline Conochilus unicornis Rousselet, 1892 & $\mathrm{X}$ & & & & & & & $\mathrm{X}$ & $\mathrm{X}$ & & $\mathrm{X}$ & $\mathrm{X}$ & & & $\mathrm{X}$ \\
\hline DICRANOPHORIDAE & & & & & & & & & & & & & & & \\
\hline Aspelta cf. angusta Harring \& Myers, 1928 & & & & & & & & & & & & & & & $\mathrm{X}$ \\
\hline *Dicranophoroides claviger (Hauer, 1965) & & $\mathrm{X}$ & & & $\mathrm{X}$ & & & & & & & & & & $\mathrm{X}$ \\
\hline Dicranophoroides caudatus (Ehrenberg, 1834) & & $\mathrm{X}$ & & & & & & & & & & & & & $\mathrm{X}$ \\
\hline $\begin{array}{l}\text { *D. caudatus brasiliensis Koste, } 1972[= \\
\text { Dicranophoroides caudatus (Ehrenberg, 1834)] }\end{array}$ & & & & & & & & & & & & & & & $\mathrm{X}$ \\
\hline Dicranophorus epicharis Harring \& Myers, 1928 & & & & & & & & & & & & $\mathrm{X}$ & & & $\mathrm{X}$ \\
\hline Dicranophorus forcipatus (Müller, 1786) & & $\mathrm{X}$ & $\mathrm{X}$ & & $\mathrm{X}$ & & & & & & & & & & $\mathrm{X}$ \\
\hline Dicranophorus grandis (Ehrenberg, 1832) & & $\mathrm{X}$ & $\mathrm{X}$ & & $\mathrm{X}$ & & & & & & & & & & \\
\hline Dicranophorus hercules Wiszniewski, 1932 & & $\mathrm{X}$ & & & & & & & & & & & & & \\
\hline $\begin{array}{l}\text { Dicranophorus kostei Pourriot \& Zoppi de Roa, } \\
1981\end{array}$ & & $X$ & & & & & & & & & & & & & \\
\hline $\begin{array}{l}\text { Dicranophorus cf. robustus Harring \& Myers, } \\
1928\end{array}$ & & & & & & & & & & & & & & & $\mathrm{X}$ \\
\hline Dicranophorus tegillus Harring \& Myers, 1928 & & $X$ & $\mathrm{X}$ & & $X$ & & & & & & & & & & $\mathrm{X}$ \\
\hline Encentrum felis (Müller, 1773) & & & & & & & & & & & & & & $\mathrm{X}$ & \\
\hline Encentrum longisepes Wulfert, 1936 & & & & & & & & & & & & & & & $\mathrm{X}$ \\
\hline Encentrum saundersiae (Hudson, 1885) & & & & & & & & & & & & & & & $\mathrm{X}$ \\
\hline $\begin{array}{l}\text { *Paradicranophorus wockei Koste, } 1961[=\mathrm{de} \\
\text { Kostea wockei (Koste, 1961)] }\end{array}$ & & & & & & & & & & & & & & & $\mathrm{X}$ \\
\hline EPIPHANIDAE & & & & & & & & & & & & & & & \\
\hline Epiphanes clavulata (Ehrenberg, 1832) & & $\mathrm{X}$ & & & & & & & & $\mathrm{X}$ & $\mathrm{X}$ & & & & $\mathrm{X}$ \\
\hline Epiphanes macroura (Barrois \& Daday, 1894) & & $X$ & & & & & & & & & & $\mathrm{X}$ & & $\mathrm{X}$ & $\mathrm{X}$ \\
\hline Mikrocodides robustus (Glascott, 1893) & & & & & & & & & & & & & & & $\mathrm{X}$ \\
\hline EUCHLANIDAE & & & & & & & & & & & & & & & \\
\hline Beauchampiella eudactylota (Gosse, 1886) & $\mathrm{X}$ & $\mathrm{X}$ & & & $\mathrm{X}$ & $\mathrm{X}$ & & $\mathrm{X}$ & & $\mathrm{X}$ & $\mathrm{X}$ & $\mathrm{X}$ & & $\mathrm{X}$ & $\mathrm{X}$ \\
\hline Dipleuchlanis propatula (Gosse, 1886$)$ & $\mathrm{X}$ & $\mathrm{X}$ & $\mathrm{X}$ & & & $\mathrm{X}$ & & $\mathrm{X}$ & $\mathrm{X}$ & $\mathrm{X}$ & $\mathrm{X}$ & $\mathrm{X}$ & & & $\mathrm{X}$ \\
\hline * D. propatula f. macrodactyla (Hauer, 1965) & & & & & & & & & & $\mathrm{X}$ & & & & & $\mathrm{X}$ \\
\hline Euchlanis deflexa (Gosse, 1851) & & & & & & & & & & & & & & & $\mathrm{X}$ \\
\hline Euchlanis dilatata Ehrenberg, 1832 & $\mathrm{X}$ & & & & & & & $\mathrm{X}$ & $\mathrm{X}$ & $\mathrm{X}$ & $\mathrm{X}$ & $\mathrm{X}$ & & $\mathrm{X}$ & $\mathrm{X}$ \\
\hline
\end{tabular}


Tab. II. Cont.

\begin{tabular}{|c|c|c|c|c|c|c|c|c|c|c|c|c|c|c|c|}
\hline Táxon & 1 & 2 & 3 & 4 & 5 & 6 & 7 & 8 & 9 & 10 & 11 & 12 & 13 & 14 & 15 \\
\hline Euchlanis dilatata dilatata Ehrenberg, 1832 & & $\mathrm{X}$ & $\mathrm{X}$ & & & & & & & & & & & & \\
\hline Euchlanis dilatata lucksiana Hauer, 1930 & & $\mathrm{X}$ & & & & & & & & & & & & & $\mathrm{X}$ \\
\hline Euchlanis incisa Carlin, 1939 & & $\mathrm{X}$ & $\mathrm{X}$ & & $\mathrm{X}$ & $\mathrm{X}$ & & & & $\mathrm{X}$ & & & & $\mathrm{X}$ & $\mathrm{X}$ \\
\hline Euchlanis incisa mucronata Ahlstrom, 1934 & & $\mathrm{X}$ & & & $\mathrm{X}$ & & & & & & & & & & $\mathrm{X}$ \\
\hline Euchlanis meneta Myers, 1930 & & $\mathrm{X}$ & $\mathrm{X}$ & & & & & & & & & & & & $\mathrm{X}$ \\
\hline Euchlanis oropha Gosse, 1887 & & & & & & & & & & & & & & & $\mathrm{X}$ \\
\hline Euchlanis triquetra Ehrenberg, 1838 & & $\mathrm{X}$ & & & $\mathrm{X}$ & & & & & & & & & & \\
\hline $\begin{array}{l}\text { *Tripleuchlanis plicata plicata (Levander, 1894) } \\
\text { [= Tripleuchlanis plicata (Levander, 1894)] }\end{array}$ & & & & & & & & & & & & & & & $\mathrm{X}$ \\
\hline \multicolumn{16}{|l|}{ FILINIIDAE } \\
\hline Filinia longiseta (Ehrenberg, 1834) & $\mathrm{X}$ & $\mathrm{X}$ & & $\mathrm{X}$ & $\mathrm{X}$ & $\mathrm{X}$ & & & & & & $\mathrm{X}$ & & & $\mathrm{X}$ \\
\hline *F. longiseta longiseta (Ehrenberg, 1834) & & $\mathrm{X}$ & & & $\mathrm{X}$ & & & & & & & & & & $\mathrm{X}$ \\
\hline *F. longiseta var. limnetica (Zacharias, 1893) & & $\mathrm{X}$ & & & $\mathrm{X}$ & & & & & & & & & & $\mathrm{X}$ \\
\hline *F. longiseta var. saltator (Gosse, 1886) & & $\mathrm{X}$ & $\mathrm{X}$ & & $\mathrm{X}$ & & & & & & & & & & $\mathrm{X}$ \\
\hline $\begin{array}{l}\text { F. cf. novaezealandiae Shiel \& Sanoamuang, } \\
1993\end{array}$ & & $\mathrm{X}$ & & & & & & & & & & & $\mathrm{X}$ & & \\
\hline F. opoliensis (Zacharias, 1898) & $\mathrm{X}$ & $\mathrm{X}$ & & $\mathrm{X}$ & $\mathrm{X}$ & $\mathrm{X}$ & & & & & & $\mathrm{X}$ & $\mathrm{X}$ & & $\mathrm{X}$ \\
\hline F. pejleri Hutchinson, 1964 & & & & & & & & & & & & & & & $\mathrm{X}$ \\
\hline F. saltator (Gosse, 1886) & & & & $\mathrm{X}$ & & & & & & & & & & & $\mathrm{X}$ \\
\hline F. terminalis (Plate, 1886) & $\mathrm{X}$ & $\mathrm{X}$ & & $\mathrm{X}$ & $\mathrm{X}$ & & & & & & & & & & $\mathrm{X}$ \\
\hline \multicolumn{16}{|l|}{ FLOSCULARIIDAE } \\
\hline Beauchampia crucigera (Dutrochet, 1812) & & $\mathrm{X}$ & $\mathrm{X}$ & & $\mathrm{X}$ & & & & & & & & & & \\
\hline Floscularia melicerta (Ehrenberg, 1832) & & & & & & & & & & & & & & & $\mathrm{X}$ \\
\hline F. pedunculata (Joliet, 1883) & & $\mathrm{X}$ & $\mathrm{X}$ & & & & & & & & & & & & \\
\hline F. ringens (Linnaeus, 1758) & & $\mathrm{X}$ & $\mathrm{X}$ & & $\mathrm{X}$ & & & & & & & & & & $\mathrm{X}$ \\
\hline Limnias ceratophylli Schrank, 1803 & & $\mathrm{X}$ & $\mathrm{X}$ & & $\mathrm{X}$ & & & & & & & & & & $\mathrm{X}$ \\
\hline L. melicerta Weisse, 1848 & & $\mathrm{X}$ & $\mathrm{X}$ & & $\mathrm{X}$ & & & & & & & & & & $\mathrm{X}$ \\
\hline *Octotrocha speciosa Thorpe, 1893 & & & $\mathrm{X}$ & & $\mathrm{X}$ & & & & & & & & & & \\
\hline Ptygura furcillata (Kellicott, 1889) & & & $\mathrm{X}$ & & & & & & & & & & & & \\
\hline P. linguata Edmondson, 1939 & & $\mathrm{X}$ & & & $\mathrm{X}$ & & & & & & & & & & \\
\hline P. longicornis (Davis, 1867) & & & $\mathrm{X}$ & & & & & & & & & & & & $\mathrm{X}$ \\
\hline P. pedunculata Edmondson, 1939 & & & & & $\mathrm{X}$ & & & & & & & & & & \\
\hline P. velata (Gosse, 1851$)$ & & & $\mathrm{X}$ & & & & & & & & & & & & \\
\hline Sinantherina ariprepes Edmondson, 1939 & & & $\mathrm{X}$ & & & & & & & & & & & & $\mathrm{X}$ \\
\hline S. procera (Thorpe, 1893) & & & & & & & & & & & & & & & $\mathrm{X}$ \\
\hline S. semibullata (Thorpe, 1893) & & & & & $\mathrm{X}$ & & & & & & & & & & \\
\hline S. socialis (Linnaeus, 1758) & $\mathrm{X}$ & & $\mathrm{X}$ & & & & & & & & & & & & \\
\hline S. spinosa (Thorpe, 1893) & & $\mathrm{X}$ & $\mathrm{X}$ & & $\mathrm{X}$ & & & & & & & & & & $\mathrm{X}$ \\
\hline \multicolumn{16}{|l|}{ GASTROPODIDAE } \\
\hline Ascomorpha cf. agilis Zacharias, 1893 & & & & & & & & & & & & & & & $\mathrm{X}$ \\
\hline Ascomorpha ecaudis Perty, 1850 & & $\mathrm{X}$ & $\mathrm{X}$ & & & & $\mathrm{X}$ & & & & & & & & $\mathrm{X}$ \\
\hline Ascomorpha klementi Hauer, 1965 & & & & & & & & & & & & & & & $\mathrm{X}$ \\
\hline Ascomorpha minima Hofsten, 1909 & & $\mathrm{X}$ & & & & & & & & & & & & & \\
\hline Ascomorpha ovalis (Bergendal, 1892) & $\mathrm{X}$ & & & & & & & & & & & & & & \\
\hline Ascomorpha saltans Bartsch, 1870 & $\mathrm{X}$ & $\mathrm{X}$ & $\mathrm{X}$ & & & & & & & & & & & & $\mathrm{X}$ \\
\hline Gastropus hyptopus (Ehrenberg, 1838) & & $\mathrm{X}$ & & & & & & & & & & & & & $\mathrm{X}$ \\
\hline Gastropus stylifer (Imhof, 1891) & & $\mathrm{X}$ & & & & & & & & & & & & & \\
\hline \multicolumn{16}{|l|}{ HEXARTHRIDAE } \\
\hline Hexarthra intermedia (Wiszniewski, 1929) & & & & $\mathrm{X}$ & & & & & & & & & & $\mathrm{X}$ & \\
\hline Hexarthra intermedia brasiliensis Hauer, 1953 & $\mathrm{X}$ & $\mathrm{X}$ & $\mathrm{X}$ & & $\mathrm{X}$ & & & & & & & $\mathrm{X}$ & & & $\mathrm{X}$ \\
\hline $\begin{array}{l}\text { Hexarthra intermedia intermedia (Wiszniewski, } \\
\text { 1929) }\end{array}$ & & $\mathrm{X}$ & $\mathrm{X}$ & & & & & & & & & & & & \\
\hline Hexarthra mira (Hudson, 1871) & & $\mathrm{X}$ & & & $\mathrm{X}$ & & & & & & & & & & $\mathrm{X}$ \\
\hline \multicolumn{16}{|l|}{ ITURIDAE } \\
\hline Itura chamadis Harring \& Myers, 1928 & & & & & & & & & & & & & & & $\mathrm{X}$ \\
\hline Itura viridis (Stenroos, 1898) & & & $\mathrm{X}$ & & $\mathrm{X}$ & & & & & & & & & & \\
\hline \multicolumn{16}{|l|}{ LECANIDAE } \\
\hline L. aculeata (Jakubski, 1912) & $\mathrm{X}$ & & & & & $\mathrm{X}$ & & $\mathrm{X}$ & & & $\mathrm{X}$ & & & & $\mathrm{X}$ \\
\hline *L. althausi Rudescu, 1960 & & & & & & $\mathrm{X}$ & & & & & & & & & \\
\hline L. amazonica (Murray, 1913) & & & & & & & & & & & & & & & $\mathrm{X}$ \\
\hline L. arcuata (Bryce, 1891) & $\mathrm{X}$ & & & & & & & & & & & & & & \\
\hline
\end{tabular}


Tab. II. Cont.

\begin{tabular}{|c|c|c|c|c|c|c|c|c|c|c|c|c|c|c|c|}
\hline Táxon & 1 & 2 & 3 & 4 & 5 & 6 & 7 & 8 & 9 & 10 & 11 & 12 & 13 & 14 & 15 \\
\hline L. bulla goniata (Harring \& Myers, 1926) & $\mathrm{X}$ & & & & & & & & & & & & & & $\mathrm{X}$ \\
\hline *Lecane bulla styrax (Harring \& Myers, 1926) & & & & & & & & & & & & & & & $\mathrm{X}$ \\
\hline L. closterocerca $($ Schmarda, 1859) & & $\mathrm{X}$ & & & $\mathrm{X}$ & $\mathrm{X}$ & & $\mathrm{X}$ & & & $\mathrm{X}$ & & & & $\mathrm{X}$ \\
\hline *L. closterocerca amazonica Koste, 1978 & & & & & & & & $\mathrm{X}$ & $\mathrm{X}$ & $\mathrm{X}$ & $\mathrm{X}$ & & & & $\mathrm{X}$ \\
\hline *L. closterocerca f. wulferti (Hauer, 1956) & & & & & $\mathrm{X}$ & & & & & & & & & & $\mathrm{X}$ \\
\hline L. copeis (Harring \& Myers, 1926) & & & & & & $\mathrm{X}$ & & & & & & & & & \\
\hline L. cornuta $($ Müller, 1786$)$ & $\mathrm{X}$ & $\mathrm{X}$ & $\mathrm{X}$ & & $\mathrm{X}$ & $\mathrm{X}$ & & & & & & & & & $\mathrm{X}$ \\
\hline *L. cornuta var. rotunda (Fadeew, 1927) & & & & & & & & & & & & & & & $\mathrm{X}$ \\
\hline L. crenata (Harring, 1913) & & $\mathrm{X}$ & $\mathrm{X}$ & & $\mathrm{X}$ & & & & & & & & & & $\mathrm{X}$ \\
\hline Lecane crepida Harring, 1914 & & & & & & & & & & & & & & & $\mathrm{X}$ \\
\hline L. curvicornis (Murray, 1913) & & $\mathrm{X}$ & $\mathrm{X}$ & & $\mathrm{X}$ & $\mathrm{X}$ & & $\mathrm{X}$ & $\mathrm{X}$ & $\mathrm{X}$ & & & & $\mathrm{X}$ & $\mathrm{X}$ \\
\hline *L. curvicornis nitida (Murray, 1913) & & & & & & & & & & $\mathrm{X}$ & & & & & $\mathrm{X}$ \\
\hline L. doryssa Harring, 1914 & $\mathrm{X}$ & & $X$ & & $\mathrm{X}$ & & & & & & & & & & $\mathrm{X}$ \\
\hline L. elegans Harring, 1914 & & & $\mathrm{X}$ & & & & & & & & & & & & $\mathrm{X}$ \\
\hline L. elsa Hauer, 1931 & & $\mathrm{X}$ & $\mathrm{X}$ & & $\mathrm{X}$ & $\mathrm{X}$ & & $\mathrm{X}$ & $\mathrm{X}$ & $\mathrm{X}$ & $\mathrm{X}$ & & & & $\mathrm{X}$ \\
\hline L. eutarsa Harring \& Myers, 1926 & & & & & $\mathrm{X}$ & & & & & & & & & & \\
\hline L. furcata (Murray, 1913) & & $\mathrm{X}$ & $\mathrm{X}$ & & $\mathrm{X}$ & $\mathrm{X}$ & & & & & & & & & \\
\hline L. galeata (Bryce, 1892) & & & $\mathrm{X}$ & & $\mathrm{X}$ & & & & & & & & & & \\
\hline L. grandis (Murray, 1913) & & & & & & & & & & & & & & & $\mathrm{X}$ \\
\hline L. haliclysta Harring \& Myers, 1926 & & & & & $\mathrm{X}$ & $\mathrm{X}$ & & & & & & & & & $\mathrm{X}$ \\
\hline L. hamata (Stokes, 1896) & $\mathrm{X}$ & $\mathrm{X}$ & $\mathrm{X}$ & & $\mathrm{X}$ & $\mathrm{X}$ & & $\mathrm{X}$ & $\mathrm{X}$ & $\mathrm{X}$ & $\mathrm{X}$ & & & $\mathrm{X}$ & $\mathrm{X}$ \\
\hline L. hastata (Murray, 1913) & & & & & & & & & & & & & & & $\mathrm{X}$ \\
\hline Lecane hornemanni (Ehrenberg, 1834) & & & & & & & & & & & & & & & $\mathrm{X}$ \\
\hline L. imbricata Carlin, 1939 & $\mathrm{X}$ & & & & & & & & & & & & & & \\
\hline L. inermis (Bryce, 1892) & & $\mathrm{X}$ & & & & & & & & & & & & & $\mathrm{X}$ \\
\hline L. inopinata Harring \& Myers, 1926 & & $\mathrm{X}$ & & & $\mathrm{X}$ & & & & & & & & & & $\mathrm{X}$ \\
\hline L. leontina (Turner, 1892) & & $\mathrm{X}$ & $\mathrm{X}$ & & $\mathrm{X}$ & $\mathrm{X}$ & $\mathrm{X}$ & $\mathrm{X}$ & $\mathrm{X}$ & $\mathrm{X}$ & $\mathrm{X}$ & & & & $\mathrm{X}$ \\
\hline L. ludwigii (Eckstein, 1883) & & & $\mathrm{X}$ & & $\mathrm{X}$ & $\mathrm{X}$ & & & $\mathrm{X}$ & $\mathrm{X}$ & $\mathrm{X}$ & & & & $\mathrm{X}$ \\
\hline *L. ludwigii ludwigii (Eckstein, 1883) & & & & & & & & & & & & & & & $\mathrm{X}$ \\
\hline *L. ludwigii f. ohioensis (Herrick 1885) & & & & & & & & & & & & & & & $\mathrm{X}$ \\
\hline L. luna (Müller, 1776) & & & & & $\mathrm{X}$ & & & $\mathrm{X}$ & $\mathrm{X}$ & $\mathrm{X}$ & $\mathrm{X}$ & & & & $\mathrm{X}$ \\
\hline L. lunaris (Ehrenberg, 1832) & & & & & $\mathrm{X}$ & $X$ & & & $\mathrm{X}$ & $\mathrm{X}$ & $\mathrm{X}$ & & & & $\mathrm{X}$ \\
\hline *L. lunaris crenata (Harring, 1913) & & & & & & & & & & & & & & & $\mathrm{X}$ \\
\hline L. marchantaria Koste \& Robertson, 1983 & & & & & & $\mathrm{X}$ & & & & & & & & & \\
\hline L. margarethae Segers, 1991 & & & $\mathrm{X}$ & & $\mathrm{X}$ & & & & & & & & & & \\
\hline L. melini Thomasson, 1953 & & $\mathrm{X}$ & & & & & & & & & & & & & \\
\hline L. monostyla (Daday, 1897) & & $\mathrm{X}$ & $\mathrm{X}$ & & & & & & & & & & & & $\mathrm{X}$ \\
\hline $\begin{array}{l}\text { *L. murrayi Hauer, } 1965 \\
{[=\text { Lecane amazonica (Murray, 1913)] }}\end{array}$ & & & & & & & & $\mathrm{X}$ & & $\mathrm{X}$ & $\mathrm{X}$ & & & & $\mathrm{X}$ \\
\hline L. nana (Murray, 1913) & & & $\mathrm{X}$ & & $\mathrm{X}$ & & & & & & & & & & \\
\hline L. obtusa (Murray, 1913) & & & & & & & & & & & & & & & $\mathrm{X}$ \\
\hline L. ohioensis (Herrick, 1885) & & & $\mathrm{X}$ & & & & & & & & & & & & \\
\hline L. papuana (Murray, 1913) & & $\mathrm{X}$ & $\mathrm{X}$ & & $\mathrm{X}$ & & $\mathrm{X}$ & & & & $\mathrm{X}$ & & & & $\mathrm{X}$ \\
\hline L. pertica Harring \& Myers, 1926 & & & $\mathrm{X}$ & & & & & & & & & & & & \\
\hline L. proiecta Hauer, 1956 & $\mathrm{X}$ & $\mathrm{X}$ & & $\mathrm{X}$ & & & & & & & $\mathrm{X}$ & & & & $\mathrm{X}$ \\
\hline L. punctata (Murray, 1913) & & & $\mathrm{X}$ & & & & & & & & & & & & \\
\hline L. pyriformis (Daday, 1905) & & $\mathrm{X}$ & $\mathrm{X}$ & & $\mathrm{X}$ & $\mathrm{X}$ & & & & & & & & & $\mathrm{X}$ \\
\hline L. quadridentata (Ehrenberg, 1832) & & $\mathrm{X}$ & & & $\mathrm{X}$ & $\mathrm{X}$ & & & & $\mathrm{X}$ & & & & $\mathrm{X}$ & $\mathrm{X}$ \\
\hline L. remanei Hauer, 1964 & & & & & & & & & & & & & & & $\mathrm{X}$ \\
\hline L. rhytida Harring \& Myers, 1926 & & $\mathrm{X}$ & $\mathrm{X}$ & & $\mathrm{X}$ & & & & & & & & & & $\mathrm{X}$ \\
\hline L. robertsonae Segers, 1993 & & & $\mathrm{X}$ & & $\mathrm{X}$ & & & & & & & & & & $\mathrm{X}$ \\
\hline L. sagula Harring \& Myers, 1926 & & & & & $\mathrm{X}$ & & & & & & & & & & \\
\hline L.scutata (Harring \& Myers, 1926) & & $\mathrm{X}$ & & & $\mathrm{X}$ & & & & $\mathrm{X}$ & & & & & & $\mathrm{X}$ \\
\hline L.signifera (Jennings, 1896) & & & & & $\mathrm{X}$ & $\mathrm{X}$ & & & & & & & & & $\mathrm{X}$ \\
\hline L.signifera ploenensis (Voigt, 1902) & & & & & & & & & & & & & & & $\mathrm{X}$ \\
\hline L. stenroosi (Meissner, 1908) & & & & & & & & & & & & & & & $\mathrm{X}$ \\
\hline L. stichaea Harring, 1913 & & & & & & & & $\mathrm{X}$ & & $\mathrm{X}$ & & & & & $\mathrm{X}$ \\
\hline $\begin{array}{l}\text { *L. submagna De Ridder, } 1960 \text { [= Lecane luna } \\
\text { (Müller, 1776)] }\end{array}$ & & & & & & & & & & & $\mathrm{X}$ & & & & $\mathrm{X}$ \\
\hline L. subtilis Harring \& Myers, 1926 & & & & & $\mathrm{X}$ & & & & & & & & & & $\mathrm{X}$ \\
\hline L. tenuiseta Harring, 1914 & & & & & & & & & & & & & & & $\mathrm{X}$ \\
\hline L. thalera (Harring \& Myers, 1926) & & & $\mathrm{X}$ & & $\mathrm{X}$ & & $\mathrm{X}$ & & & & & & & & \\
\hline L. theinemanni (Hauer, 1938) & & $\mathrm{X}$ & $\mathrm{X}$ & & & & & & & & & & & & $\mathrm{X}$ \\
\hline L. undulata Hauer, 1938 & & & & & $\mathrm{X}$ & & & & & & & & & & \\
\hline L. unguitata (Fadeev, 1925) & & & & & & & & & & & & & & & $\mathrm{X}$ \\
\hline L. ungulata (Gosse, 1887) & & $\mathrm{X}$ & $\mathrm{X}$ & & & $\mathrm{X}$ & & & & & & & & & $\mathrm{X}$ \\
\hline
\end{tabular}


Tab. II. Cont.

\begin{tabular}{|c|c|c|c|c|c|c|c|c|c|c|c|c|c|c|c|}
\hline Táxon & 1 & 2 & 3 & 4 & 5 & 6 & 7 & 8 & 9 & 10 & 11 & 12 & 13 & 14 & 15 \\
\hline $\begin{array}{l}\text { *L. wulferti Hauer, } 1956[=\text { Lecane closterocerca } \\
\text { (Schmarda, 1859)] }\end{array}$ & & & & & & & & & & & & & & & $\mathrm{X}$ \\
\hline LEPADELLIDAE & & & & & & & & & & & & & & & \\
\hline Colurella obtusa (Gosse, 1886) & & $\mathrm{X}$ & & & $\mathrm{X}$ & $\mathrm{X}$ & & & & & & & & & $\mathrm{X}$ \\
\hline Colurella cf. salina Althaus, 1957 & & $\mathrm{X}$ & & & & & & & & & & & & & \\
\hline Colurella uncinata (Müller, 1773) & & & & & $\mathrm{X}$ & $\mathrm{X}$ & & & & & & & & & \\
\hline Colurella cf. uncinata & & & & & & & & & & & & & & $\mathrm{X}$ & \\
\hline Colurella uncinata bicuspidata (Ehrenberg, 1832) & & $\mathrm{X}$ & & & $\mathrm{X}$ & & & & & & & & & & \\
\hline Lepadella acuminata (Ehrenberg, 1834) & & & & & & & & & & & & & & & $\mathrm{X}$ \\
\hline L. benjamini Harring, 1916 & & & & & & & & & & $\mathrm{X}$ & $\mathrm{X}$ & & & & $\mathrm{X}$ \\
\hline L. cristata (Rousselet, 1893) & & & $\mathrm{X}$ & & & & & & & & $\mathrm{X}$ & & & & $\mathrm{X}$ \\
\hline L. dactyliseta (Stenroos, 1898) & & $X$ & & & & & & & & & & & & & \\
\hline L. donneri Koste, 1972 & & $\mathrm{X}$ & $\mathrm{X}$ & & $\mathrm{X}$ & & & & & $\mathrm{X}$ & $\mathrm{X}$ & & & & $\mathrm{X}$ \\
\hline *L. ehrenbergi (Perty, 1850) & & & & & $\mathrm{X}$ & & & & & & & & & & \\
\hline *L. heterodactyla Fadeew, 1925 & & & & & $\mathrm{X}$ & & & & & & & & & & \\
\hline L. latusinus (Hilgendorf, 1899) & & $\mathrm{X}$ & $\mathrm{X}$ & & $\mathrm{X}$ & $\mathrm{X}$ & & & & & & & & & \\
\hline L. ovalis (Müller, 1786) & & $\mathrm{X}$ & & & $\mathrm{X}$ & $\mathrm{X}$ & & & & & & & & $\mathrm{X}$ & $\mathrm{X}$ \\
\hline L. patella (Müller, 1773) & & $\mathrm{X}$ & & & & $\mathrm{X}$ & & & & $\mathrm{X}$ & $\mathrm{X}$ & & & & \\
\hline L. pterygoida (Dunlop, 1897) & & & & & & $\mathrm{X}$ & & & & & & & & & \\
\hline L. quadricarinata (Stenroos, 1898) & & & & & $\mathrm{X}$ & & & & & & & & & & \\
\hline L. quinquecostata (Lucks, 1912) & & & & & & & & & & & & & & & $\mathrm{X}$ \\
\hline L. rhomboides (Gosse, 1886) & & $\mathrm{X}$ & $\mathrm{X}$ & & $\mathrm{X}$ & $\mathrm{X}$ & & & & & & & & & $\mathrm{X}$ \\
\hline L. rottenburgi (Lucks, 1912) & $\mathrm{X}$ & & & & & & & & & & & & & & \\
\hline L. triba Myers, 1934 & & $X$ & & & & & & & & & & & & & \\
\hline L. triptera (Ehrenberg, 1832) & & & & & $\mathrm{X}$ & & & & & & & & & & $\mathrm{X}$ \\
\hline LINDIIDAE & & & & & & & & & & & & & & & \\
\hline Lindia truncata (Jennings, 1894) & & & $\mathrm{X}$ & & & & & & & & & & & & \\
\hline MYTILINIDAE & & & & & & & & & & & & & & & \\
\hline Lophocharis oxysternon (Gosse, 1851) & & & & & & & & & & & & & & & $\mathrm{X}$ \\
\hline Lophocaris salpina (Ehrenberg, 1834) & & & & & & $X$ & & & & & & & & & \\
\hline Mytilina acanthophora Hauer, 1938 & & & & & & & & & & & & & & & $\mathrm{X}$ \\
\hline Mytilina bisulcata (Lucks, 1912) & & & & & $\mathrm{X}$ & & $\mathrm{X}$ & & & & & & & & \\
\hline Mytilina crassipes (Lucks, 1912) & & & & & $\mathrm{X}$ & & & & & & & & & & \\
\hline Mytilina macrocera (Jennings, 1894) & & & & & & & & & & & & & & & $\mathrm{X}$ \\
\hline Mytilina mucronata (Müller, 1773) & & & & & & & & & & & & & & & $\mathrm{X}$ \\
\hline Mytilina mucronata spinigera (Ehrenberg, 1830) & & & & & & & & & & & & & & & $\mathrm{X}$ \\
\hline Mytilina trigona (Gosse, 1851$)$ & & $\mathrm{X}$ & & & & $\mathrm{X}$ & & & & & & & & & $\mathrm{X}$ \\
\hline M. cf. unguipes (Lucks, 1912) & & & & & & & & & & & & & & & $\mathrm{X}$ \\
\hline Mytilina ventralis (Ehrenberg, 1830) & & & & & & $\mathrm{X}$ & & & & $\mathrm{X}$ & & & & $\mathrm{X}$ & $\mathrm{X}$ \\
\hline Mytilina ventralis brevispina (Ehrenberg, 1830) & & & & & & & & & & & & & & & $\mathrm{X}$ \\
\hline * Mytilina ventralis macracantha (Gosse, 1886$)$ & & $\mathrm{X}$ & $\mathrm{X}$ & & $\mathrm{X}$ & & & & & & & & & & $\mathrm{X}$ \\
\hline Mytilina ventralis ventralis (Ehrenberg, 1830) & & & & & $\mathrm{X}$ & & & & & & & & & & \\
\hline NOTOMMATIDAE & & & & & & & & & & & & & & & \\
\hline Cephalodella anebodica Bērziņš, 1976 & & & & & & & & & & & & & & & $\mathrm{X}$ \\
\hline C. forficula (Ehrenberg, 1830) & & & & & $\mathrm{X}$ & & & & & & & & & & $\mathrm{X}$ \\
\hline C. gibba (Ehrenberg, 1830) & & & $\mathrm{X}$ & & $\mathrm{X}$ & & & & & & & & & & $\mathrm{X}$ \\
\hline C. gigantea Remane, 1933 & & $\mathrm{X}$ & $\mathrm{X}$ & & & & & & & & & & & & \\
\hline C. gracilis (Ehrenberg, 1830) & & & & & $\mathrm{X}$ & & & & & & & & & & \\
\hline C. stenroosi Wulfert, 1937 & & & & & & & & & & & & & & & $\mathrm{X}$ \\
\hline C. sterea (Gosse, 1887$)$ & & $\mathrm{X}$ & & & $\mathrm{X}$ & & & & & & & & & & $\mathrm{X}$ \\
\hline C. tenuiseta (Burn, 1890) & & $\mathrm{X}$ & & & & & & & & & & & & & $\mathrm{X}$ \\
\hline Enteroplea lacustris Ehrenberg, 1830 & & & & & & & & & & & & & & & $\mathrm{X}$ \\
\hline Eosphora anthadis Harring \& Myers, 1922 & & & $\mathrm{X}$ & & & & & & & & & & & & \\
\hline E. thoides Wulfert, 1935 & & & & & $\mathrm{X}$ & & & & & & & & & & \\
\hline Monommata actices Myers, 1930 & & & & & $\mathrm{X}$ & & & & & & & & & & \\
\hline M. enedra Myers, 1930 & & & & & & & & & & & & & & & $\mathrm{X}$ \\
\hline M. cf. longiseta (Müller, 1786) & & & & & & & & & & & & & & & $\mathrm{X}$ \\
\hline M. maculata Harring \& Myers, 1930 & & $\mathrm{X}$ & $\mathrm{X}$ & & $\mathrm{X}$ & & & & & & & & & & $\mathrm{X}$ \\
\hline Notommata allantois Wulfert, 1935 & & & $\mathrm{X}$ & & $\mathrm{X}$ & & & & & & & & & & \\
\hline N. cerberus (Gosse, 1886) & & $\mathrm{X}$ & & & & & & & & & & & & & \\
\hline N. copeus Ehrenberg, 1834 & & $\mathrm{X}$ & & & $\mathrm{X}$ & & & & & & & & & & $\mathrm{X}$ \\
\hline N. cyrtopus Gosse, 1886 & & & & & $\mathrm{X}$ & & & & & & & & & & \\
\hline N. falcinella Harring \& Myers, 1922 & & & & & & & & & & & & & & & $\mathrm{X}$ \\
\hline N. glyphura Wulfert, 1935 & & $\mathrm{X}$ & & & $\mathrm{X}$ & & & & & & & & & & $\mathrm{X}$ \\
\hline N. haueri Wulfert, 1939 & & & & & & & & & & & & & & & $\mathrm{X}$ \\
\hline N. pachyura (Gosse, 1886$)$ & & $\mathrm{X}$ & & & $\mathrm{X}$ & & & & & & & & & & \\
\hline
\end{tabular}


Tab. II. Cont.

\begin{tabular}{|c|c|c|c|c|c|c|c|c|c|c|c|c|c|c|c|}
\hline Táxon & 1 & 2 & 3 & 4 & 5 & 6 & 7 & 8 & 9 & 10 & 11 & 12 & 13 & 14 & 15 \\
\hline N. prodota Myers, 1933 & & & & & & & & & & & & & & & $\mathrm{X}$ \\
\hline N. tripus Ehrenberg, 1838 & & & & & & & & & & & & & & & $\mathrm{X}$ \\
\hline Taphrocampa selenura Gosse, 1887 & & $\mathrm{X}$ & $\mathrm{X}$ & & & & & & & & & & & & \\
\hline SCARIDIIDAE & & & & & & & & & & & & & & & \\
\hline Scaridium longicaudum (Müller, 1786) & & & $\mathrm{X}$ & & $\mathrm{X}$ & $\mathrm{X}$ & & & & & & $\mathrm{X}$ & & & $\mathrm{X}$ \\
\hline SYNCHAETIDAE & & & & & & & & & & & & & & & \\
\hline *Ploesoma africana Wulfert, 1961 & & & & & $\mathrm{X}$ & & & & & & & & & & \\
\hline P. lenticulare Herrick, 1885 & & & & & & & & & & & & & & & $\mathrm{X}$ \\
\hline P. truncatum (Levander, 1894) & & $\mathrm{X}$ & & & & & & & & & & & & & $\mathrm{X}$ \\
\hline Polyarthea dolichoptera Idelson, 1925 & $\mathrm{X}$ & & & & $\mathrm{X}$ & $\mathrm{X}$ & & & & & & & & $\mathrm{X}$ & $\mathrm{X}$ \\
\hline P. vulgaris Carlin, 1943 & & $\mathrm{X}$ & $\mathrm{X}$ & & $\mathrm{X}$ & & & & $\mathrm{X}$ & & & $\mathrm{X}$ & & $\mathrm{X}$ & $\mathrm{X}$ \\
\hline $\begin{array}{l}\text { *P. vulgaris var. longiremis Koste, } 1978 \text { (= } \\
\text { Polyarthra longiremis Carlin, 1943) }\end{array}$ & $\mathrm{X}$ & & & & & & & & & & & & & & \\
\hline P. remata Skorikov, 1896 & & $\mathrm{X}$ & & & $\mathrm{X}$ & & & & & & & & & & $\mathrm{X}$ \\
\hline Synchaeta grandis Zacharias, 1893 & & & & & $\mathrm{X}$ & & $\mathrm{X}$ & & & & & & & & \\
\hline S. littoralis Rousselet, 1902 & & & & & $\mathrm{X}$ & & & & & & & & & & \\
\hline S. longipes Gosse, 1887 & & $\mathrm{X}$ & & & & & & & & & & & & & $\mathrm{X}$ \\
\hline S. pectinata Ehrenberg, 1832 & & $\mathrm{X}$ & & $\mathrm{X}$ & $\mathrm{X}$ & & & & & & & & & & $\mathrm{X}$ \\
\hline S. stylata Wierzejski, 1893 & $\mathrm{X}$ & $\mathrm{X}$ & & & & & & & & & & & & & $\mathrm{X}$ \\
\hline TESTUDINELLIDAE & & & & & & & & & & & & & & & \\
\hline Pompholyx complanata Gosse, 1851 & & $\mathrm{X}$ & & & $\mathrm{X}$ & & & & & & & & & & \\
\hline P. cf. sulcata Hudson, 1885 & & & & & & & & & & & & & & $\mathrm{X}$ & \\
\hline P. cf. triloba Pejler, 1957 & & & & & & & & & & & & & & & $\mathrm{X}$ \\
\hline Testudinella ahlstromi Hauer, 1956 & & $\mathrm{X}$ & & & $\mathrm{X}$ & & $\mathrm{X}$ & & & & & & & & $\mathrm{X}$ \\
\hline T. amphora Hauer, 1938 & & $\mathrm{X}$ & & & $\mathrm{X}$ & & & & & & & & & & \\
\hline T. cf. brevicaudata Yamamoto, 1951 & & & $\mathrm{X}$ & & $\mathrm{X}$ & & & & & & & & & & \\
\hline T. emarginula (Stenroos, 1898) & & $\mathrm{X}$ & & & $\mathrm{X}$ & & & & & & & & & & \\
\hline T. greeni Koste, 1981 & & & & & & & & & & & & & & & $\mathrm{X}$ \\
\hline T. hauerensis Gillard, 1967 & & & $\mathrm{X}$ & & $\mathrm{X}$ & & & & & & & & & & \\
\hline T. mucronata (Gosse, 1886) & & & & & $\mathrm{X}$ & $\mathrm{X}$ & & & & & & & & & \\
\hline *T. mucronata hauerensis (Gillard, 1967) & & & & & & & & & & & $\mathrm{X}$ & & & & $\mathrm{X}$ \\
\hline T. ohlei Koste, 1972 & & & & & & & & & & & & & & & $\mathrm{X}$ \\
\hline T. patina (Hermann, 1783) & $\mathrm{X}$ & $\mathrm{X}$ & $\mathrm{X}$ & & $\mathrm{X}$ & $\mathrm{X}$ & & $\mathrm{X}$ & $\mathrm{X}$ & $\mathrm{X}$ & $\mathrm{X}$ & $\mathrm{X}$ & & $\mathrm{X}$ & $\mathrm{X}$ \\
\hline *T. patina f. intermedia Anderson, 1889 & & & & & & & & $\mathrm{X}$ & & & & & & & $\mathrm{X}$ \\
\hline T. tridentata Smirnov, 1931 & & & & & $\mathrm{X}$ & & & & & & & & & & \\
\hline *T. tridentata amazonica Thomasson, 1971 & & & & & & & & & & & & & & & $\mathrm{X}$ \\
\hline TETRASIPHONIDAE & & & & & & & & & & & & & & & \\
\hline Tetrasiphon hydrocora Ehrenberg, 1840 & & & & & & & & & & & & & & & $\mathrm{X}$ \\
\hline TRICHOCERCIDAE & & & & & & & & & & & & & & & \\
\hline Trichocerca bicristata (Gosse, 1887) & & $\mathrm{X}$ & $\mathrm{X}$ & & $\mathrm{X}$ & $\mathrm{X}$ & & $\mathrm{X}$ & & $\mathrm{X}$ & $\mathrm{X}$ & $\mathrm{X}$ & & & $\mathrm{X}$ \\
\hline *T. bicristata var. mucosa (Stokes, 1896) & & & & & & & & & & $\mathrm{X}$ & & & & & \\
\hline T. bidens (Lucks, 1912) & & & & & $\mathrm{X}$ & & & $\mathrm{X}$ & & & & & & & $\mathrm{X}$ \\
\hline T. cf. brachyura (Gosse, 1851) & & & & & & & & & & & & & & $\mathrm{X}$ & \\
\hline T. braziliensis (Murray, 1913) & $\mathrm{X}$ & $\mathrm{X}$ & $\mathrm{X}$ & & $\mathrm{X}$ & & & & & $\mathrm{X}$ & $\mathrm{X}$ & & & & $\mathrm{X}$ \\
\hline T. capucina (Wierzejski \& Zacharias, 1893) & & & & & & & & & & $\mathrm{X}$ & & & & & $\mathrm{X}$ \\
\hline T. chattoni (de Beauchamp, 1907) & $\mathrm{X}$ & $X$ & & & $\mathrm{X}$ & & & & & & & & & & \\
\hline T. cylindrica (Imhof, 1891) & & & & & $\mathrm{X}$ & & & & & & & & & & $\mathrm{X}$ \\
\hline T. cylindrica chattoni (de Beauchamp, 1907) & & & & & & & & & $\mathrm{X}$ & & & & & & $\mathrm{X}$ \\
\hline T. dixon-nuttali (Jennings, 1903) & & & & & & & & & & & & & & & $\mathrm{X}$ \\
\hline T. elongata (Gosse, 1886$)$ & & & & & & & & & & & $\mathrm{X}$ & $\mathrm{X}$ & & & $\mathrm{X}$ \\
\hline T. flagellata Hauer, 1937 & & & & & & & & & & $\mathrm{X}$ & & & & & \\
\hline T. gracilis $($ Tessin, 1890$)$ & $\mathrm{X}$ & & & & & & & & & & & & & & $\mathrm{X}$ \\
\hline T. heterodactyla (Tschugunoff, 1921) & & & & & & & & & & & & & & & $\mathrm{X}$ \\
\hline T. iernis (Gosse, 1887) & & & & & & & & & & & & & & & $\mathrm{X}$ \\
\hline T. insignis (Herrick, 1885) & & & $\mathrm{X}$ & & $\mathrm{X}$ & & & & $\mathrm{X}$ & $\mathrm{X}$ & & & & & $\mathrm{X}$ \\
\hline T. longiseta (Schrank, 1802) & $\mathrm{X}$ & & $\mathrm{X}$ & & $\mathrm{X}$ & & & & & & & & & & $\mathrm{X}$ \\
\hline T. macera (Gosse, 1886$)$ & & & & & & & & & & & & & & & $\mathrm{X}$ \\
\hline T. myersi (Hauer, 1931) & & & & & & & & & & & & & & & $\mathrm{X}$ \\
\hline T. plaka Myers, 1938 & & & & & & & & & & & & & & & $\mathrm{X}$ \\
\hline T. porcellus (Gosse, 1851) & & & & & & & & & & & & & & & $\mathrm{X}$ \\
\hline T. pusilla (Jennings, 1903) & & $\mathrm{X}$ & $\mathrm{X}$ & & & & & & & & & & & & $\mathrm{X}$ \\
\hline T. rattus (Müller, 1776) & & & & & & $\mathrm{X}$ & & & $\mathrm{X}$ & $\mathrm{X}$ & & & & & \\
\hline T. rousseleti (Voigt, 1902) & & $\mathrm{X}$ & & & & & & & & & & & & & $\mathrm{X}$ \\
\hline T. ruttneri Donner, 1953 & & $\mathrm{X}$ & & & & & & & & & & & & & $\mathrm{X}$ \\
\hline T. scipio (Gosse, 1886) & & & & & & & & & & & & & & & $\mathrm{X}$ \\
\hline T. similis (Wierzejski, 1893) & $\mathrm{X}$ & & & & $\mathrm{X}$ & $\mathrm{X}$ & & & & & & $\mathrm{X}$ & & & $\mathrm{X}$ \\
\hline
\end{tabular}


Tab. II. Cont

\begin{tabular}{|c|c|c|c|c|c|c|c|c|c|c|c|c|c|c|c|}
\hline Táxon & 1 & 2 & 3 & 4 & 5 & 6 & 7 & 8 & 9 & 10 & 11 & 12 & 13 & 14 & 15 \\
\hline T. similis grandis Hauer, 1965 & & & & & & & & & & & & & & & $\mathrm{X}$ \\
\hline T. similis similis (Wierzejski, 1893) & & $\mathrm{X}$ & & & $\mathrm{X}$ & & & & & & & & & & \\
\hline T. stylata (Gosse, 1851) & $\mathrm{X}$ & & & & & & & & $\mathrm{X}$ & $\mathrm{X}$ & & & & & $\mathrm{X}$ \\
\hline T. tenuior (Gosse, 1886) & & $\mathrm{X}$ & $\mathrm{X}$ & & $\mathrm{X}$ & & & & & & & & & & \\
\hline T. tigris (Müller, 1786) & & $\mathrm{X}$ & $\mathrm{X}$ & & $\mathrm{X}$ & & & & & & & & & & $\mathrm{X}$ \\
\hline TRICHOTRIIDAE & & & & & & & & & & & & & & & \\
\hline Macrochaetus collinsi (Gosse, 1867) & & $\mathrm{X}$ & $\mathrm{X}$ & & $\mathrm{X}$ & $\mathrm{X}$ & & & & & & & & & $\mathrm{X}$ \\
\hline M. longipes Myers, 1934 & & & & & & & & & & & & & & & $\mathrm{X}$ \\
\hline M. multispinosus Myers, 1934 & & $\mathrm{X}$ & & & $\mathrm{X}$ & & & & & & & & & & \\
\hline M. sericus (Thorpe, 1893) & $\mathrm{X}$ & & & & & & & & & $\mathrm{X}$ & $\mathrm{X}$ & $\mathrm{X}$ & & & $\mathrm{X}$ \\
\hline M. subquadratus (Perty, 1850) & & & & & & & & & & & & & & & $\mathrm{X}$ \\
\hline Trichotria tetractis (Ehrenberg, 1830) & & & & & & & & $\mathrm{X}$ & $\mathrm{X}$ & & & $\mathrm{X}$ & & & $\mathrm{X}$ \\
\hline T. tetractis similis (Stenroos, 1898) & & $\mathrm{X}$ & $\mathrm{X}$ & & $\mathrm{X}$ & & & & & & & & & & \\
\hline TROCHOSPHAERIDAE & & & & & & & & & & & & & & & \\
\hline Horaella brehmi Donner, 1949 & $\mathrm{X}$ & & & & & & & & & & & & & & \\
\hline H. thomassoni Koste, 1973 & & & & & & & & & & & & & & & $\mathrm{X}$ \\
\hline Trochosphaera aequatorialis Semper, 1872 & $\mathrm{X}$ & & & & & & & & & & & & & & $\mathrm{X}$ \\
\hline Riqueza de táxons & 51 & 154 & 98 & 17 & 148 & 52 & 9 & 21 & 20 & 37 & 33 & 31 & 10 & 27 & 244 \\
\hline
\end{tabular}

deste equipamento é um obstáculo aos estudos taxonômicos de Rotifera no Brasil.

O conhecimento sobre a taxonomia morfológica devese aliar à ecologia e taxonomia molecular para aumentar a precisão dos diagnósticos. Silva \& Matsumura-Tundisi (2005) sugeriram marcadores moleculares, ITS2 (Espaço transcrito interno 2) para Copepoda como auxílio a taxonomia tradicional. Esta abordagem pode ser também útil para os cladóceros (ABREU et al., 2010). Em relação aos rotíferos, estudos desta categoria demonstraram que existem espécies crípticas em várias morfoespécies, como, por exemplo, Brachionus calyciflorus (GILBERT \& WALSH, 2005). Mais estudos deste tipo são necessários no Brasil.

Um fator que dificulta a taxonomia dos rotíferos é o alto grau de plasticidade fenotípico em morfologia de vários gêneros, como Brachionus e Keratella (Pejler, 1977), com a descrição de elevados números de subespécies, formas e variedades, de relações taxonômicas confusas. Assim, características utilizadas na taxonomia podem variar em relação às características do ambiente, como temperatura e predadores (SANOAMUANG, 1993a; GILBERT, 2011). Estudos espaço-temporais de biometria de populações destas formas podem ser importantes no esclarecimento de suas posições taxonômicas (Roche, 1993; GIRI \& JosÉ DE PAGGI, 2006).

Finalmente, a necessidade de chaves de identificação em português [como da chave de José DE PAGGi (1995) em espanhol] é grande; a chave mais comumente usada ainda é de Koste (1978) que, além de antiquada, é na língua alemã. As chaves mais atualizadas de SEgERs (1995), NogRady et al. (1995), De Smet (1996), De Smet \& Pourriot (1997), Ricci \& Melone (2000), e Nogrady \& Segers (2002) são em inglês, e assim mais acessível. Porém, uma chave no modelo de El Moor-Loureiro (1997) para Cladocera seria útil em estudos taxonômicos de Rotifera no Brasil.

Agradecimentos. A Fundação de Apoio ao Desenvolvimento do Ensino, Ciências e Tecnologia do Estado de Mato Grosso do Sul (FUNDECT) e a Superintendência de Ciências e Tecnologia do Estado de Mato Grosso do Sul (SUCITEC/MS) pelo convite de participação neste fascículo especial da Iheringia, Série Zoologia e o suporte financeiro para sua publicação.

\section{REFERÊNCIAS BIBLIOGRÁFICAS}

Abreu, M. J.; Santos-Winiewski, M. J.; Rocha, O. \& Orlando, T. C. 2010. The use of PCR-RFLP to genetically distinguish the morphologically close species: Ceriodaphnia dubia Richard, 1894 and Ceriodaphnia silvestrii Daday, 1902 (Crustacea Cladocera). Brazilian Journal of Biology 70:121-124.

Bonecker, C. C.; Aoyagui, A. S. M. \& Santos, R. M. 2009. The impact of impoundment on the rotifer communities in two floodplain environments: interannual pulse variations. Brazilian Journal of Biology 69:529-537.

Bonecker, C. C.; Lansac-TôHa, F. A. \& Bini, L. M. 1998. Composition of zooplankton communities in different environments of the Mato Grosso Pantanal, Mato Grosso, Brazil. In: Anais do VIII Seminário Regional de Ecologia, v. III, p. 1123-1135.

Bonecker, C. C.; LansaC-TôHa, F. A. \& Staub, A. 1994. Qualitative study of rotifers in different environments of the High Paraná River floodplain (MS) - Brazil. Revista UNIMAR 16:1-16.

CAlheiros, D. F. 2003. Influência do pulso de inundação na composição isotópica $\left(\delta^{13} \mathrm{C}\right.$ e $\left.\delta^{15} \mathrm{~N}\right)$ das fontes primárias de energia na planície de inundação do rio Paraguai, Pantanal - MS. Tese de doutorado. Piracicaba, Centro de Energia Nuclear na Agricultura, Universidade de São Paulo. 164p.

Clément, P. \& Wurdak, E. 1991. Rotifera. In: Harrison, F. W. \& RupPert, E. E. eds. Microscopic Anatomy of Invertebrates 4: Aschelminthes. New York, Wiley-Liss, p. 219-297.

De SMet, W. H. 1998. Preparation of rotifer trophi for light and scanning electron microscopy. Hydrobiologia 387,388:117-121.

De Smet, W. H. 1996. The Proalidae (Monogononta). Rotifera 4. In: DuMONT, H. J. ed. Guides to the identification of microinvertebrates of continental waters of the world 9. The Netherlands, SPB Academic. $344 p$.

De Smet, W. H. 2005. Study of the trophi of Testudinella Bory de St. Vincent and Pompholyx Gosse (Rotifera: Testudinellidae) by scanning electron microscopy. Hydrobiologia 546:203-211.

De Smet, W. H. \& Pourriot, R. 1997. The Dicranophoridae and: The Ituridae (Monogononta). Rotifera 5. In: Dumont, H. J. ed. Guides to the identification of microinvertebrates of continental waters of the world 12. The Netherlands, SPB Academic. 344p.

El Moor Loureiro, L. M. A. 1997. Manual de identificação de cladóceros límnicos do Brasil. Brasília, Editora Universa-UCB. 156p.

Espíndola, E. G.; Matsumura-Tundisi, T. \& Moreno, I. H. 1996. Efeitos da dinâmica hidrológica do sistema Pantanal Matogrossense sobre a estrutura da comunidade de zooplancton da Lagoa Albuquerque. Acta Limnologica Brasiliensia 8:37-57.

Fontaneto, D.; Barbosa, A. M.; Segers, H. \& Pautasso, M. 2012. The 'rotiferologist' effect and other global correlates of species richness in monogonont rotifers. Ecography 35:174-182. 
Frutos, S. M.; Poi De Neiff, A. S. G. \& NeifF, J. J. 2006. Zooplankton of the Paraguay River: a comparison between sections and hydrological phases. Annales de Limnologie - International Journal of Limnology 42:277-288.

Garcia, A. P. P.; Lansac-Tôha, F. A. \& Bonecker, C. C. 1998. Species composition and abundance of rotifers in different environments of the floodplain of the upper Paraná River, Brazil. Revista Brasileira de Zoologia 15:327-343.

Garraffoni, A. R. S. \& LourençO, A. P. 2012. Synthesis of Brazilian Rotifera: An updated list of species. Check List 8:375-407.

GILBERT, J. J. 2011. Induction of different defences by two enemies in the rotifer Keratella tropica: response priority and sensitivity to enemy density. Freshwater Biology 56:926-938.

Gilbert, J. J. \& Walsh, E. J. 2005. Brachionus calyciflorus is a species complex: Mating behavior and genetic differentiation among four geographically isolated strains. Hydrobiologia 546:257-265.

GIRI, F. \& JosÉ DE PAGGI, S. 2006. Geometric morphometric and biometric analysis for the systematic elucidation of Brachionus caudatus Barrois and Daday, 1894 (Rotifera Monogononta Brachionidae) forms. Zoologischer Anzeiger 244:171-180.

ICZN. INTERNATIONAL COMMISSION ON ZOOLOGICAL NOMECLATURE. 1999. International Code of Zoological Nomenclature. London, The International Trust for Zoological Nomenclature. 306p.

Joko, C. Y.; Lansac-TôHa, F. A.; Murakami, E. A. \& Bonecker, C. C. 2008. Novas ocorrências de Lecane no plâncton de distintos ambientes da planície de inundação do alto rio Paraná. Acta Scientiarum, Biological Sciences 30:165-171.

José de PAggi, S. 1995. Rotifera. In: Lopretto, E. C. \& Tell. G. eds. Ecosistemas de aguas continentales. Metodologias para su estudio. II. La Plata, Ediciones Sur, p.643-667.

Koste, W. 1978. Rotatoria. Die Rädertiere Mitteleuropas. Berlin, Gebrüder Borntraeger. 2 vol.

Koste, W. 1999. Uber Radertiere (Rotifera) aus Gewassern des sudlichen Pantanal (Brasilien). Osnabrücker Naturwissenschaftliche Mitteilungen 25:179-209.

LansaC-TôHa, F. A.; Bonecker, C. C.; Velho, L. F. M. \& Lima, A. F. 1997. Composição, distribuição e abundância da comunidade zooplanctônica. In: Vazzoler, A. E. A. M.; Agostinho, A. A. \& Hahn, N. S. eds. A planície de inundação do alto rio Paraná. Maringá, Editora UEM, p.117-155.

LansaC-Tôha, F. A.; Bonecker, C. C.; Velho, L. F. M.; Simões, N. R.; Dias, J. D.; Alves, G. M. \& Takahashi, E. M. 2009. Biodiversity of zooplankton communities in the Upper Paraná River floodplain: interannual variation from long-term studies. Brazilian Journal of Biology 69:539-549.

Markevich, G. I. \& Kutikova, L. A. 1989. Mastax morphology under SEM and its usefulness in reconstructing rotifer phylogeny and systematics. Hydrobiologia 186,187:285-289.

Martínez, J. C. C.; Canesin, A. \& Bonecker, C. C. 2000. Species composition of rotifers in different habitats of an artificial lake, Mato Grosso do Sul State, Brazil. Acta Scientiarum 22:343-346.

MEDINA-JÚNIOR, P. B. 1999. Estrutura e diversidade da comunidade zooplanctônica em lagoas naturais do Pantanal de Nhecolândia - MS. Dissertação de Mestrado. São Carlos, Ciências da Engenharia Ambiental, Universidade de São Paulo.

Nogrady, T. \& SEgErs, H. 2002. Asplanchnidae, Filiniidae, Gastropodidae, Lindiidae, Microcodidae and Synchaetidae. Rotifera 6. In: Dumont, H. J. ed. Guides to the identification of microinvertebrates of continental waters of the world 18. The Netherlands, Backhuys Publishers BV. 264p.

Nogrady, T. \& Rowe, T. L. A. 1993. Comparative laboratory studies of narcosis in Brachionus plicatilis. Hydrobiologia 255,256:51-56.
Nogrady, T.; Pourriot, R. \& Segers, H. 1995. The Notommatidae and the Scaridiidae. Rotifera 3. In: Dumont, H. J. ed. Guides to the identification of microinvertebrates of continental waters of the world 8. The Netherlands, SPB Academic. 248p.

Obertegger, U.; Braioni, M. G.; Arrighett, G. \& Flaim, G. 2006. Trophi morphology and its usefulness for identification of formalin-preserved species of Synchaeta Ehrenberg, 1832 (Rotifera: Monogononta: Synchaetidae). Zoologischer Anzeiger 245:109-120.

Oliveira-Neto, A. L. 1991. Rotifers from Pantanal. A flood area in Brazil. End of Course Report of the International training course on Lake zooplankton: a tool in lake management. Belgium, State University of Ghent, Laboratory of Limnology.

Oliveira-Neto, A. L. \& Moreno, I. H. 1999. Rotíferos. In: Joly, C. A. \& Bicudo, C. E. M. coord. Biodiversidade do Estado de São Paulo, Brasil: síntese do conhecimento ao final do século XX. Invertebrados de água doce. São Paulo, FAPESP, v.4.

Ricci, C. \& Melone, G. 2000. Key to the identification of the genera of bdelloid rotifers. Hydrobiologia 418:73-80.

Roche, K. F. 1993. Temporal variation in the morphology of the rotifer Keratella quadrata (Müller, 1786). Annales de Limnologie 29:119-127.

Roche, K. F. 2010. Estudo da eutrofização de uma pequena represa urbana ("Lago do Amor", Campo Grande, MS): qualidade da água, biomonitoramento, e modelagem. Relatório Técnico. Campo Grande, Fundect/UFMS.

SANOAMUANG, L. 1993a. The effect of temperature on morphology, life history and growth rate of Filinia terminalis (Plate) and Filinia cf. pejleri Hutchinson in culture. Freshwater Biology 30:257-267.

SANOAMUANG, L. 1993b. Comparative studies on scanning electron microscopy of trophi of the genus Filinia Bory De St. Vincent (Rotifera). Hydrobiologia 264:115-128.

Segers, H. 1995. The Lecanidae (Monogononta). Rotifera 2. . In: Dumont, H. J. ed. Guides to the identification of microinvertebrates of continental waters of the world 6 . The Netherlands, SBP Academic. 226 p.

Segers, H. 2007. Annotated checklist of the rotifers (Phylum Rotifera), with notes on nomenclature, taxonomy and distribution. Auckland, New Zealand, Magnolia Press. 104p.

SEGERS, H. 2008. Global diversity of rotifers (Rotifera) in freshwater. Hydrobiologia 595:49-59.

Segers, H.; Koste, W. \& Yussef, S. M. 1996. Contribution to the knowledge of the monogonont Rotifera of Zanzibar, with a note on Filinia novaezealandiae Shiel \& Sanoamuang. Internationale Revue der gesamten Hydrobiologie 96:597-603.

Serafim, M. Jr.; Bonecker, C. C.; Rossa, D.C.; LansaC-TôHa, F. A. \& Costa, C. L. 2003. Rotifers of the upper Paraná River floodplain: additions to the checklist. Brazilian Journal of Biology 63:207-212.

SHIEL, R. J. \& SANOAMUANG, L. 1993. Trans-Tasman variation in Australasian Filinia populations. Hydrobiologia 255,256:455-462.

SiLva, W. M. \& MATSUmurA-TundisI, T. 2005. DNA extraction and ITS2 (Internal Transcribed Spacer 2) gene sequences of some Brazilian freshwater copepods. Verhandlungen des Internationalen Verein Limnologie 29:409-413.

Silva, W. M.; Roche, K. F. \& Rocha, O. 2010. Zooplankton communities of hyposaline Pantanal lakes in Brazil. Verhandlungen des Internationalen Verein Limnologie 30:1530-1530.

Souza-Soares, F.; Tundisi, J. G. \& Matsumura-Tundisi, T. M. 2011. Checklist de Rotifera de água doce do Estado de São Paulo, Brasil. Biota Neotropica 11(1a):1-25. Disponível em: $<$ http://www.biotaneotropica. org.br/v11n1a/pt/fullpaper?bn0231101a2011+pt ${ }^{\text {. }}$.

Wallace, R. L.; Snell, T. W.; Ricci, C. \& Nogrady, T. 2006. Rotifera Biology, ecology and systematics. 2ed. Holanda, Kenobi Productions; Gent, Belgica, Backhuys Academic Publishing B. V. 299 